\title{
Propensity scores: Methods, considerations, and applications in the Journal of Thoracic and Cardiovascular Surgery
}

\author{
Timothy L. McMurry, PhD, ${ }^{a}$ Yinin Hu, MD, ${ }^{b}$ Eugene H. Blackstone, MD, ${ }^{c}$ and \\ Benjamin D. Kozower, MD, MPH ${ }^{\mathrm{a}, \mathrm{b}}$
}

\begin{abstract}
Objective: To review the published literature using propensity scoring, describe shortcomings in the use of this technique, and provide conceptual background for understanding and correctly implementing studies that use propensity matching.

Methods: We survey the published statistical literature and make recommendations for a set of standard criteria for studies that use propensity matching. We evaluated adherence to these criteria in recent publications in the Journal of Thoracic and Cardiovascular Surgery and determined how well the standards were applied.
\end{abstract}

Results: We found that studies that use propensity matching are rarely documented well enough to be convincing in their results. When documentation is available, statistical shortcomings are common.

Conclusions: Improved statistical practice is needed when using propensity scoring. This article suggests standard criteria for using this method in Journal publications. (J Thorac Cardiovasc Surg 2015;150:14-9)

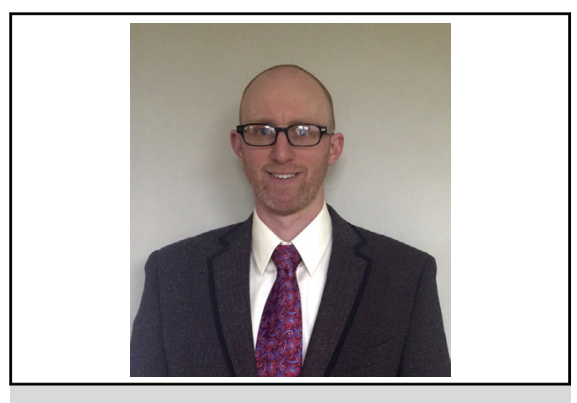

Timothy L. McMurry, PhD.

\section{Central Message}

We provide conceptual background information and good-practice recommendations for use of propensity matching in surgical outcomes research.

\begin{abstract}
Perspective
The frequent use of propensity matching requires that it be understood by clinicians and researchers. Retrospective studies comparing 2 treatments are hampered by selection bias; propensity scoring reduces the bias by selecting similar groups for comparison. Review of recent JTCVS publications suggests that the methods are not consistently applied. This article highlights the important concepts.
\end{abstract}

See Editorial Commentary page 20 .
Propensity scoring is a powerful tool to strengthen causal inferences drawn from observational studies. The motivation is simple: To compare the effects of 2 treatment options, which we generically refer to as " $\mathrm{A}$ " and "B," with $\mathrm{B}$ being the more common one, we want to compare the outcomes of similar groups of patients receiving each

From the ${ }^{\mathrm{a}}$ Department of Public Heath Sciences, University of Virginia; ${ }^{\mathrm{b}}$ Division of Thoracic Surgery, Department of Surgery, University of Virginia Health System, Charlottesville, Va; and ${ }^{c}$ Department of Thoracic and Cardiovascular Surgery and Department of Quantitative Health Sciences, Research Institute, Cleveland Clinic, Cleveland, Ohio.

Received for publication March 16, 2015; accepted for publication March 20, 2015; available ahead of print May 9, 2015.

Address for reprints: Benjamin D. Kozower, MD, MPH, University of Virginia Health System, General Thoracic Surgery, PO Box 800679, Charlottesville, VA 229080679 (E-mail: bdk8g@virginia.edu).

0022-5223/\$36.00

Copyright (c) 2015 by The American Association for Thoracic Surgery

http://dx.doi.org/10.1016/j.jtcvs.2015.03.057 treatment. Propensity scoring helps in selecting similar patient groups for comparison.

Propensity scoring is common in the literature, and the methodology is widely discussed. ${ }^{1-8}$ Despite the popularity of propensity scoring, we are concerned that its use is conceptually more intricate than many investigators realize. The consequence can be results that are misleading, or difficult for readers, referees, and investigators to evaluate objectively. These concerns persist, despite the fact that they have been raised previously in the cardiothoracic surgery literature. ${ }^{9}$ The problem is compounded by inconsistent recommendations in the methodologic literature (see later section: Analysis of the Matched Data).

In the present article, we review the basics of propensity scoring, highlight areas of general agreement and disagreement on practical recommendations, discuss choices available to the investigator, examine how this family of techniques has been applied in recent Journal of 
Thoracic and Cardiovascular Surgery (JTCVS) articles, and establish guidelines for future articles. Conceptual background is provided, followed by general guidelines, and finally, examination of recent articles published in JTCVS.

\section{THE CONCEPTUAL FRAMEWORK FOR PROPENSITY MATCHING}

Many medical studies are designed to evaluate the effectiveness of a treatment in comparison to an alternative. The goal is to estimate how much better (or worse) the outcomes are for patients in 1 treatment group, compared with what would have happened had they received the other treatment. Such comparisons need to account for differences in patients that may have contributed to their allocation to 1 of the treatments.

The randomized controlled trial, in which patients are randomly assigned to treatment groups, is the "gold standard" for this comparison. The importance of random assignment is that the patient characteristics affecting outcomes (eg, age, gender, comorbidities, mental disposition) tend to be equally distributed across groups. Thus, significant differences in outcomes can be attributed to the treatment.

However, many questions cannot be addressed by a randomized trial, owing to cost, ethical or practical considerations, and timeliness. For example, in a study of smoking-related healthcare costs, patients cannot ethically (or practically) be randomly assigned to smoke or not smoke. ${ }^{6}$ Moreover, a randomized study would take decades to run. Other variables of interest cannot be assigned; Koch and colleagues ${ }^{10}$ considered whether men and women fare differently after coronary artery bypass grafting. Many of these clinical questions can be addressed with robust observational data.

Unfortunately, in observational data, patients receiving treatment A typically differ systematically from those receiving $\mathrm{B}$, leaving direct comparison of outcomes heavily confounded. For example, patients who receive surgery are deemed well enough before treatment to survive the surgery, whereas extremely frail patients may be deemed inoperable. Comparing outcomes between such dissimilar patients is not fair or appropriate.

Historically, investigators tried to account for differences between groups by using multiple regression to adjust for confounding characteristics. However, because the groups of patients may differ systematically, using a regression to estimate the potential effect of treatment $\mathrm{A}$ on a patient who received treatment $\mathrm{B}$ can be an unreliable extrapolation. Therefore, limiting comparison to only those patients who are legitimate candidates for either procedure is imperative.

An intuitively appealing approach would be to match patients from 1 treatment group with patients from the other on several important characteristics, and compare their outcomes. Unfortunately, matching on even a modest number of criteria often leaves a large majority of patients unmatched and unavailable for analysis, making the results less reliable. ${ }^{11}$

Propensity scores solve the problem of matching on multiple covariates by reducing them to a single quantity, the propensity score. A patient's propensity score is defined as the probability that the patient receives treatment A (instead of B), given all relevant conditions, comorbidities, and other characteristics at the time the treatment decision is made. What makes propensity scores so powerful is that, under some conditions, patients with the same propensity score have the same probabilistic distribution of other covariates, regardless of whether they received treatment $\mathrm{A}$ or $\mathrm{B}{ }^{12}$ As a result, a sufficient analysis can be a comparison of outcomes, across treatment groups, of pairs or pools of patients with similar propensity scores.

\section{STEPS IN A PROPENSITY-SCORE ANALYSIS}

An analysis using propensity scores has 4 main steps. First, the propensity scores must be estimated (later section: Constructing the Propensity-Score Model). Second, the data need to be matched or grouped based on the estimated propensity scores (later section: Grouping the Data). Third, balance must be assessed, to ensure that the grouping produced similar pools of patients receiving treatment $\mathrm{A}$ versus B (later section: Assessment of Covariate Balance in Matched Groups). Finally, data can be analyzed to estimate the treatment effect size and its clinical and statistical significance (later section: Analysis of the Matched Data).

The first 3 of these are "design" steps, used to frame a comparison around similar groups of patients; they must be performed without looking at the outcomes data. None of the steps can be adequately performed by following a simple recipe. However, before tackling these technical issues, 2 crucial assumptions must be met for propensity matching to provide useful results.

\section{CRUCIAL ASSUMPTIONS}

Two conditions on the data must be met for analyses based on propensity scores to provide valid results. The most important condition is known as "strong ignorability," which means that the treatment assignment (A or B) is independent of a patient's potential outcomes under the 2 treatment scenarios, given the covariates. In other words, the observed covariates contain all the information about the patient's condition that is relevant to potential outcomes. Strong ignorability makes intuitive sense. If the goal is to compare similar groups of patients receiving different treatments, we need to know all the factors that determine whether patients are comparable at the time of treatment allocation. 
The second condition is that, given the covariates, the patient needs to have a positive probability of receiving both treatments. Intuitively, this condition can be understood as the idea that there is no gain in asking what the potential benefits of surgery are for a patient whose comorbidities preclude survival of an operation. The only interest is in comparing patients for whom either treatment is realistic.

\section{CONSTRUCTING THE PROPENSITY-SCORE MODEL}

The first step is to estimate the propensity scores for each patient. The most common approach is to use logistic regression, but other regression models can estimate classification probabilities. ${ }^{13,14}$

\section{Which Variables to Include}

Guidelines for constructing and evaluating a regression model depend on its intended application. ${ }^{15}$ For propensity scores, the "strong ignorability" condition necessitates inclusion of covariates that predict potential outcomes under either treatment scenario, as well as any covariates that predict treatment assignment, although these 2 criteria are typically related. From a practical point of view, the second of these requirements deserves particular emphasis: If the data do not contain the information used to make the treatment decision (or are systematically missing in 1 of the 2 patient groups), the propensity model will be inadequate, and all subsequent analyses will be suspect.

The consensus is that if the sample size is too small for the propensity-score model to include all variables of interest, the most important to include are variables that are strongly related to outcome. ${ }^{16}$ These should be selected a priori, based on scientific understanding and previous literature, and without reference to the outcomes within the dataset. ${ }^{17}$ Having too many predictors is probably better than having too few, ${ }^{7}$ and when sample sizes are large, good propensity models can contain many predictors. ${ }^{6}$

\section{Logistic Regression-Model Diagnostics}

The goal of the propensity-score model is to create balanced groups of patients receiving each treatment. Therefore, some model-evaluation tools, such as those evaluating discriminative ability (eg, the $c$-statistic), multicollinearity, and model selection, are of only secondary importance. ${ }^{18}$ The crucial diagnostic step is to compare covariate balance between the resulting 2 groups of patients, each receiving 1 of the 2 treatments (see later section: Assessment of Covariate Balance in Matched Groups).

A model that accurately estimates the likelihood of treatment allocation is the key to achieving this balance. Nonetheless, some common metrics in many regression applications are less important in the present context. For example, multicollinearity occurs when highly correlated predictors produce instability in their corresponding coefficients. Fortunately, multicollinearity does not affect the resulting fitted values, in this case, propensity scores. However, if the sample size is limited, it may still be advantageous to remove highly correlated variables in order to include less correlated covariates.

In addition, concern has been raised about traditional model-selection strategies, such as stepwise variable selection. These approaches are designed for prediction rather than covariate balance. The concern is that these selection methods might remove variables that are weakly related to treatment assignment, but strongly related to outcome, ${ }^{16}$ even though variables related to outcome are considered at least as important.

The commonly used $c$-statistic requires nuanced interpretation in this setting. In most applications, a predictive model with a low $c$-statistic is useless. A propensity model with a low $c$-statistic could be caused by poor construction; however, it could also be indicative of differences in practice that are not related to patient condition. The former problem invalidates subsequent analyses, whereas the latter can be beneficial. For example, imagine trying to estimate propensity scores for a randomized trial. A well constructed model accounting for all relevant clinical covariates has a $c$-statistic of approximately 0.5 , and all patients should have similar propensity scores. Nonetheless, a randomized trial is ideally suited for causal inference. At the other extreme, a $c$-statistic close to 1 indicates that the regression model is able to differentiate patients receiving treatment $\mathrm{A}$ from those receiving treatment $\mathrm{B}$, indicating that the 2 groups may be so different that their outcomes are difficult to compare meaningfully.

\section{GROUPING THE DATA}

Once propensity scores have been estimated, the data are typically grouped by either subclassification (sometimes called stratification) or matching. Both of these methods prune the original dataset down to groups or sets of patients with similar propensity scores. While there are other approaches, we focus on these 2 for their simplicity and frequency of use.

Before grouping, it may be reasonable to remove patients receiving 1 treatment who have propensity scores that are either much larger or much smaller than any patient receiving the other treatment- the "oranges," as discussed in Blackstone. ${ }^{2}$ The rationale for exclusion is that these patients do not seem to have been candidates for the alternative treatment. Nonetheless, excluded patients should be examined carefully. If many patients are unmatchable, the propensity-score model may include a variable that is a strong surrogate for treatment assignment, 
which may be removed. ${ }^{2}$ In addition, evaluation of the "oranges" will help reveal the limits within which a valid comparison of the 2 treatments is possible.

\section{Subclassification}

Subclassification is frequently suggested in the methodologic literature but less frequently applied. The idea is simple: propensity scores are grouped, eg, into quintiles or deciles (5-10 groups is typical). ${ }^{19}$ Within each group, the propensity scores are similar, so grouped patients should have similar covariate distributions, and thus can be compared. An analysis is performed in each group, and results are aggregated. Subclassification has intuitive appeal because it focuses comparisons on pools of patients with similar propensity scores. In contrast, if patients are matched, many matches may be suitable for a group-A patient, with some potential matches arbitrarily excluded from final comparisons.

\section{Matching}

The more common approach is to match individual patients receiving 1 treatment to patients with similar propensity scores receiving the other. Although conceptually simple, the details lead to different algorithms, which can affect subsequent analyses. These variations include the methods for measuring distances between propensity scores, the threshold for what constitutes matching scores, how 1 match is chosen from many candidates, the number of patients in group B (the larger group) matched to each patient in group A, and whether a single patient in group $\mathrm{B}$ can be matched to more than 1 individual in group A.

Intuition suggests that the distance between propensity scores should be measured by the simple difference between estimated probabilities of treatment. This approach is commonly used; however, evidence indicates that it is more effective to match on the "linear propensity score," or the difference between propensity scores on the logit scale. ${ }^{20}$

A decision must be made on how close 2 propensity scores need to be before they can be potential matches; this threshold is known as a "caliper." A narrow caliper can prevent inaccurate matching, but if too many patients go unmatched, the results can become uninterpretable. The appropriate caliper size depends on the relative variations of propensity scores in the 2 treatment groups. ${ }^{20,21}$

Next, the user must decide how many patients in group B should be matched to each patient in group A. The most common approach is to match each patient in group A to a single patient in group B. If group B is much larger than group A, matching a larger number of patients in group B may be advantageous, but the benefits are reduced if the extra matches are of poor quality. Finally, reuse of patients in group B is a possibility. This method makes the matching process independent of the order in which the matches are selected and may improve the overall match quality.
However, without adjustment, reused patients have too much weight in the final comparison of outcomes.

Once these decisions have been made, pairing is often done using a "greedy" algorithm. The group A patients are randomly ordered. The first of these randomly ordered patients is matched to their best group-B counterpart. The group-B patient is removed from the set of potential future matches, and the process is repeated. An alternative to "greedy" matching is "optimal" matching, ${ }^{4}$ which seems to produce better matched pairs, but does not substantially improve the balance of the matched groups as a whole. ${ }^{22}$ Stuart $^{23}$ maintains a web page describing available software.

\section{ASSESSMENT OF COVARIATE BALANCE IN MATCHED GROUPS}

Given that the goal in using propensity scores is to create pools of similar patients for comparison, assessment of the postmatching similarity across groups is extremely important to complete, before any assessment of outcomes. In particular, all covariates affecting patients' prognoses before treatment, and indications for treatment, need to be compared. If clinically relevant differences remain after matching, subsequent analyses are unreliable.

The types of comparisons differ depending on how the data are grouped. If the data are subclassified, then one should perform diagnostics within each subclass. If the data are matched, then typically comparison is between the matched pools of patients.

Most investigators assess covariate balance using hypothesis tests. For example, an investigator might test the hypothesis that the average age of patients in group A is the same as their matched counterparts in group B. Unfortunately, hypothesis tests answer the wrong question. The $P$ value from a hypothesis test depends on the difference between the 2 groups and their sample sizes. However, only the difference between the 2 groups is relevant to covariate balance. ${ }^{24}$

A better metric for continuous covariates is a measure that does not depend on sample size, such as the standardized difference in means ${ }^{20}:\left(\bar{X}_{\mathrm{A}}-\bar{X}_{\mathrm{B}}\right) / \sigma_{\mathrm{A}}$, which expresses the difference between the 2 groups in standard deviations. The improvement in balance achieved by matching can be demonstrated by comparing standardized differences in means before and after matching (using the same estimate for $\sigma_{\mathrm{A}}$ in both quantities). Binary covariates can be compared with a simple difference in proportions, or by a similar standardized difference. ${ }^{25}$ Alternatively, Rubin suggests a set of powerful, but less intuitive, diagnostics. ${ }^{17}$

\section{ANALYSIS OF THE MATCHED DATA}

The final step in a propensity-score analysis is to estimate the treatment effect size and its clinical and statistical 
significance. Literature on the proper analysis of matched data is sparse and occasionally in conflict. For example, Rosenbaum $^{4}$ recommends analyzing the data with permutation tests in the same way one would analyze an unmatched observational trial. Austin ${ }^{26}$ argues that propensity-matched data should be analyzed using procedures for matched analyses, such as paired $t$ tests, and McNemar's test. Stuart ${ }^{27}$ replies that matched analyses are not necessary, and that the data can be analyzed using a standard regression that includes a treatment indicator and the variables used in the matching. A recent article by $\mathrm{Li}$ and Greene ${ }^{28}$ suggests that a weighting method is optimal. Many articles make almost no mention of statistical inference.

This confusion has resulted because statistical understanding is still evolving, and assumptions made about the data and matching process can alter the estimates' derived properties. In most cases relevant to surgical outcomes, regression is defensible and even recommendable. Propensity scores provide an objective way to restrict the domain of analysis to patients who are legitimate candidates for either procedure. Outcomes in the 2 groups are then compared using a regression model that controls for all covariates used in matching, plus a treatment indicator variable. The coefficient associated with this indicator is interpreted as the treatment effect. An advantage of regression is that it provides some level of "double robustness" by adjusting for any remaining small covariate imbalances. ${ }^{29}$ For this reason, even randomized trials are sometimes analyzed with regression models.

Regression is more important following subclassification because, within subclasses, meaningful covariate imbalances may remain. In this setting, recommendations for the regression remain similar. If enough data are available, a regression model containing the treatment indicator and all covariates can be fit in each subclass, and the results combined. If data are more limited, a single regression model may be fit containing subclass indicators and subclass-by-treatment interactions, along with the other covariates. This approach keeps the covariate relationships fixed but allows different size treatment effects across the subclasses. After regression modeling, subclass-specific treatment effects are then combined by a weighted average of the treatment effects in each subclass, with the effects typically weighted by the number of group-A individuals in the subclass.

\section{INTERPRETATION}

For matched data, patients receiving treatment $\mathrm{A}$ have been grouped with a probabilistically similar pool of group- $B$ patients. Therefore, the estimated effect size represents the average improvement of the group-A patients relative to similar patients in group B. This quantity is traditionally described in the literature as the average treatment effect in the treated, which is not the same as the average effect of treatment across the entire population, referred to as the average treatment effect. In most cases, we suspect that the average treatment effect in the treated is the desired quantity, as it describes the benefits and risks of treatment A relative to those for similar patients receiving treatment $\mathrm{B}$, rather than as a potential benefit averaged across all patients.

Both measures assume that all group-A patients in the initial dataset were included in the final analyzed groups. If many patients have been excluded, the interpretation may change, or results may become uninterpretable; see earlier section: Grouping the Data.

\section{RECOMMENDATIONS FOR PUBLISHED LITERATURE}

Although we recognize the importance of brevity, propensity-scoring methods must be described well enough that results can be evaluated and replicated. Most of our recommendations can be implemented with 1 or 2 paragraphs. In some cases, additional tables may be provided in online appendices.

Although different analyses are appropriate for different datasets and clinical questions, we propose that articles on studies utilizing propensity matching include the following:

1. The original sample sizes for the pools of patients in each group.

2. The sample sizes available after matching.

3. The type of regression model used to estimate the propensity scores.

4. The variables considered for inclusion in the propensity model, the variables included in the final model, and the inclusion criteria.

5. The type of matching algorithm used.

6. Diagnostics demonstrating the quality of the resulting matches.

7. Characterization of the unmatched patients.

8. An indication of the statistical procedures used for analyses.

\section{JOURNAL OF THORACIC AND CARDIOVASCULAR SURGERY LITERATURE REVIEW}

We reviewed all publications in JTCVS from 2013 and 2014 using propensity-score matching. We found 25 such articles in 2013, and 64 in 2014. Although many of these articles were well done, some exhibited substantial statistical shortcomings, and many did not provide enough detail for objective evaluation. Our results are summarized in Table 1. Notably, many articles showed evidence of inadequate covariate balance after matching, and no article carefully evaluated the excluded patients. 
TABLE 1. Characteristics of 2013-2014 JTCVS papers using propensity scores

\begin{tabular}{lc}
\hline \multicolumn{1}{c}{ Criteria } & $\begin{array}{c}\text { Articles that provided } \\
\text { information, } \mathbf{n}(\%)\end{array}$ \\
\hline Sample size for original dataset & $87(98)$ \\
Matched sample size & $81(91)$ \\
Type of regression model used to estimate the & $79(89)$ \\
propensity score & \\
Matching algorithm & $60(67)$ \\
Analysis of covariate balance & $66(74)$ \\
$\quad$ Evidence of inadequate covariate balance & $17(26)$ \\
$\quad$ (of the 66) & \\
Comparison of matched to unmatched patients & $0(0)$ \\
Type of statistical procedure & \\
$\quad$ Univariate, independent samples & $59(66)$ \\
$\quad$ Univariate, paired & $11(12)$ \\
$\quad$ Regression after matching & $31(35)$ \\
Regression including the propensity score as & $10(11)$ \\
$\quad$ a covariate & \\
\hline Values for n are out of a total of 89 articles, unless otherwise indicated
\end{tabular}

\section{CONCLUSIONS}

Propensity matching is a powerful tool for observational data analyses because it facilitates the comparison of outcomes between similar groups of patients. Although propensity matching has become a popular technique, the methodology is actually quite complex. This review is intended to help surgeons understand the concepts behind propensity matching that may influence their own research and/or help them critically evaluate the published literature. We identified 8 criteria that we feel should be reported in any article that uses propensity matching. When we applied these criteria to the publications in JTCVS from 2013 and 2014, concerns were raised about the use of this methodology and appropriateness of the applications. We recommend that the Journal adopt these criteria to create a standard for future articles submitted to JTCVS reporting on studies that use propensity matching.

\section{Conflict of Interest Statement}

Authors have nothing to disclose with regard to commercial support.

\section{References}

1. Blackstone EH. Breaking down barriers: helpful breakthrough statistical methods you need to understand better. J Thorac Cardiovasc Surg. 2001;122:430-9.

2. Blackstone EH. Comparing apples and oranges. J Thorac Cardiovasc Surg. 2002; 123:8-15.

3. Brookhart MA, Wyss R, Layton JB, Sturmer T. Propensity score methods for confounding control in nonexperimental research. Circ Cardiovasc Qual Outcomes. 2013;6:604-11.
4. Rosenbaum PR. Observational Studies. 2nd ed. New York: Springer; 2002.

5. Rubin D. Matched Sampling for Causal Effects, 10. New York: Cambridge University Press; 2006:12

6. Rubin DB. The design versus the analysis of observational studies for causal ef fects: parallels with the design of randomized trials. Stat Med. 2007;26:20-36.

7. Stuart EA. Matching methods for causal inference: a review and a look forward. Stat Sci. 2010;25:1-21

8. d'Agostino RB. Tutorial in biostatistics: propensity score methods for bias reduction in the comparison of a treatment to a non-randomized control group. Stat Med. 1998; 17:2265-81.

9. Austin PC. Propensity-score matching in the cardiovascular surgery literature from 2004 to 2006: a systematic review and suggestions for improvement. $J$ Thorac Cardiovasc Surg. 2007;134:1128-35.e3.

10. Koch CG, Khandwala F, Nussmeier N, Blackstone EH. Gender and outcomes after coronary artery bypass grafting: a propensity-matched comparison. J Thorac Cardiovasc Surg. 2003;126:2032-43.

11. Rosenbaum PR, Rubin DB. The bias due to incomplete matching. Biometrics 1985;41:103-16.

12. Rosenbaum PR, Rubin DB. The central role of the propensity score in observational studies for causal effects. Biometrika. 1983;70:41-55.

13. Lee BK, Lessler J, Stuart EA. Improving propensity score weighting using machine learning. Stat Med. 2010;29:337-46.

14. Westreich D, Lessler J, Funk MJ. Propensity score estimation: neural networks, support vector machines, decision trees (CART), and meta-classifiers as alternatives to logistic regression. J Clin Epidemiol. 2010;63:826-33.

15. Vittinghoff E, Shiboski S, McCulloch CE. Regression Methods in Biostatistics. 2nd ed. New York: Springer; 2010.

16. Brookhart MA, Schneeweiss S, Rothman KJ, Glynn RJ, Avorn J, Sturmer T. Variable selection for propensity score models. Am J Epidemiol. 2006;163:1149-56.

17. Rubin DB. Using propensity scores to help design observational studies: application to the tobacco litigation. Health Serv Outcomes Res. 2001;2:169-88.

18. Rubin DB. On principles for modeling propensity scores in medical research. Pharmacoepidemiol Drug Saf. 2004;13:855-7.

19. Rosenbaum PR, Rubin DB. Reducing bias in observational studies using subclassification on the propensity score. J Am Stat Assoc. 1984;79:516-24.

20. Rosenbaum PR, Rubin DB. Constructing a control group using multivariate matched sampling methods that incorporate the propensity score. Am Statis 1985;39:33-8.

21. Cochran WG, Rubin DB. Controlling bias in observational studies: a review. Sankhyā: Indian J Stat, Ser A. 1973;35:417-46.

22. Gu XS, Rosenbaum PR. Comparison of multivariate matching methods: structures, distances, and algorithms. J Comput Graphic Stat. 1993;2:405-20.

23. Stuart E. Software for implementing matching methods and propensity scores. Available at: http://www.biostat.jhsph.edu/ estuart/propensityscoresoftware html. Accessed September 5, 2014.

24. Imai K, King G, Stuart EA. Misunderstandings between experimentalists and observationalists about causal inference. J Roy Stat Soc: Ser A. 2008;171:481-502.

25. Austin PC. Using the standardized difference to compare the prevalence of a binary variable between two groups in observational research. Commun Stat Simul Comput. 2009;38:1228-34.

26. Austin PC. A critical appraisal of propensity-score matching in the medical literature between 1996 and 2003. Stat Med. 2008;27:2037-49.

27. Stuart EA. Developing practical recommendations for the use of propensity scores: Discussion of 'A critical appraisal of propensity score matching in the medical literature between 1996 and 2003' by Peter Austin, Statistics in Medicine. Stat Med. 2008;27:2062-5.

28. Li L, Greene T. A weighting analogue to pair matching in propensity score analysis. Int J Biostat. 2013;9:215-34.

29. Rubin DB. The use of matched sampling and regression adjustment to remove bias in observational studies. Biometrics. 1973;29:185-203.

Key Words: propensity score, matching, causal inference, observational studies, statistical methods 